October 20, 2015 10:49 WSPC/INSTRUCTION FILE

ws-ijmpcs*_*Capua.v3

International Journal of Modern Physics: Conference Series

2 (C) The Authors

\title{
Top quark studies with the ATLAS detector
}

\author{
Marcella Capua on behalf of the ATLAS Collaboration \\ Università della Calabria and INFN \\ Arcavacata di Rende (CS), 87036, Italy \\ marcella.capua@fis.unical.it
}

\begin{abstract}
The latest top quark studies in proton-proton collisions at a centre-of-mass energy of 7 and $8 \mathrm{TeV}$ with the ATLAS detector are reported. We present recent results on the top pair production inclusive cross-sections, top pair production differential cross-section in the resolved and boosted regimes, single top-quark production cross-sections measured in the t-channel, s-channel and W-boson associated processes, as well as the determination of the CKM matrix element $\left|V_{t b}\right|$. The results are compared with theoretical expectations. Latest ATLAS results on top properties will be also shown in terms of direct and pole mass, spin correlations and charge asymmetry.
\end{abstract}

Keywords: Top quark; ATLAS; QCD.

PACS numbers:

\section{Introduction}

The large data samples collected by the ATLAS and CMS Collaborations in $p-p$ collisions at the CERN Large Hadron Collider (LHC) at the centre-of-mass energies $\sqrt{s}=7 \mathrm{TeV}$ and $\sqrt{s}=8 \mathrm{TeV}$, allow one to study in detail the properties of the top quark and to perform precise measurements of the inclusive and differential cross-sections for top production.

The understanding of the production processes and properties of top quark is fundamental for testing the predictions of the Standard Model (SM) and for searching for signals of new physics. The top quark is the heaviest known elementary particle and due to its large mass it decays before hadronizing; therefore, all properties of the top quark are preserved in the decay chain and propagated to its decay products. Furthermore, its large coupling to the Higgs boson suggests that it might be closely connected to electroweak symmetry breaking (see e.g. Ref.1).

In this contribution an overview of the most significant results, obtained by the ATLAS Collaboration, on top quark properties and production mechanisms are presented.

This is an Open Access article published by World Scientific Publishing Company. It is distributed under the terms of the Creative Commons Attribution 3.0 (CC-BY) License. Further distribution of this work is permitted, provided the original work is properly cited. 


\section{1. Top-pair production}

${ }_{36}$ At the LHC, the top quarks are predominantly produced in pairs via the strong ${ }_{37}$ interaction and through the gluon-gluon fusion process with only a small contribu38 tion from quark-antiquark annihilation. The precise prediction of the correspond39 ing inclusive and differential cross-sections is a challenge for perturbative Quantum 40 Cromodynamics (QCD) calculations. Precise measurements of these observables are ${ }_{41}$ sensitive to the gluon parton distribustion function (PDF), to the top quark mass and to potential enhancements due to physics beyond the SM.

Within the SM, the quark top decays almost exclusively into a $\mathrm{W}$ boson and a b quark, so the final-state topology of a $t \bar{t}$ event is governed by the decay modes of the two W gauge bosons; in particular, the final states are subdivided into the dilepton channel with both $\mathrm{W}$ bosons decaying leptonically, the lepton + jets channel in which one of the $\mathrm{W}$ bosons decays leptonically and the other one hadronically, and the all hadronic channel with both $\mathrm{W}$ bosons decaying hadronically.

In this section the most recent results on inclusive and differential $t \bar{t}$ crosssections are presented; for the differential cross-sections the results are presented both in the resolved and boosted regimes.

\subsection{Inclusive top-pair cross-section measurements}

Recent ATLAS and CMS inclusive top-pair measurements using events in the dilepton channel ${ }^{2}$ and lepton+jets channel ${ }^{3}$ are summarized in figure 1 right, the results are also presented as a function of the centre-of-mass energy and compared to NNLO+NNLL QCD predictions ${ }^{4,5}$ (see figure 1 left).

The inclusive $t \bar{t}$ cross-section in the dilepton channel, $t \bar{t} \rightarrow e \mu \nu \bar{\nu} b \bar{b}$, have been measured with the data sets collected at $\sqrt{s}=7 \mathrm{TeV}$ and $\sqrt{s}=8 \mathrm{TeV}$. The analysis requires one electron and one muon with $p_{\mathrm{T}}>25 \mathrm{GeV}$ and a pseudorapidity ${ }^{\mathrm{a}}$ $\eta<2.5$. The numbers of events with exactly one and exactly two b-tagged jets were counted and used to simultaneously determine $\sigma_{t \bar{t}}$ and the efficiency to reconstruct and b-tag a jet from a top quark decay, thereby minimising the associated systematic uncertainties.

The total inclusive $t \bar{t}$ cross-sections are measured to be $\sigma_{t \bar{t}}=182.9 \pm 3.9 \% \mathrm{pb}$ and $\sigma_{t \bar{t}}=242.4 \pm 4.3 \% \mathrm{pb}$ at $\sqrt{s}=7 \mathrm{TeV}$ and $\sqrt{s}=8 \mathrm{TeV}$, respectively. The results 6 are consistent with recent NNLO+NNLL QCD calculations ${ }^{4-10}$. The inclusive crosssections were also measured in a fiducial phase-space region to reduce the systematic uncertainties associated with the acceptance estimation ${ }^{2}$.

The measurement of the total and fiducial $t \bar{t}$ cross-sections at $\sqrt{s}=8 \mathrm{TeV}$ in the lepton+jets final state used a likelihood discriminant fit method. The inclusive

aTLAS uses a right-handed coordinate system with its origin at the nominal interaction point (IP) in the centre of the detector and the $z$-axis along the beam pipe. The $x$-axis points from the IP to the centre of the LHC ring, and the $y$-axis points upward. Cylindrical coordinates $(r, \phi)$ are used in the transverse plane, $\phi$ being the azimuthal angle around the beam pipe. The pseudorapidity is defined in terms of the polar angle $\theta$ as $\eta=-\ln \tan (\theta / 2)$. 
October 20,

ws-ijmpcs*_*Capua.v3
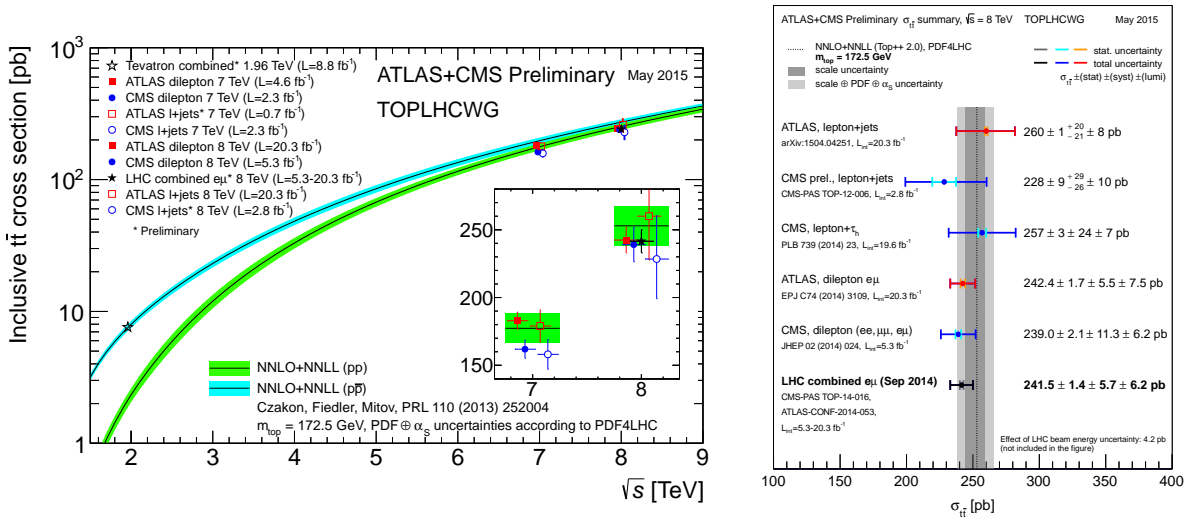

Fig. 1. (Left) Summary of the LHC and TeVatron measurements of the top-pair production cross-sections ${ }^{11}$ as a function of the centre-of-mass energy compared to the NNLO+NNLL QCD calculations. (Right) Compilation of the measurements of the top-pair production cross-sections at $8 \mathrm{TeV}$ compared to the NNLO+NNLL QCD calculations.

$t \bar{t}$ production cross-section is found to be

$$
\sigma_{t \bar{t}}=258 \pm 1(\text { stat. })_{-22}^{+21}(\text { syst. }) \pm 8(\text { lumi. }) \pm 4(\text { beam }) \mathrm{pb} .
$$

${ }_{69}$ The fiducial $t \bar{t}$ cross-section is found to be $\sigma_{t \bar{t}}^{f i d}=22.8 \pm 0.1$ (stat. $)_{-1.9}^{+1.8}($ syst. $) \pm$ 7 (lumi. $) \pm 4$ (beam) pb. The uncertainties of the measurement take into account statistical, experimental and theoretical uncertainties, the knowledge of the integrated luminosity and the LHC beam energy.

An impressive experimental precision is reached by the LHC measurements. The experimental precision matches the precision reached by the NNLO+NNLL QCD calculations ${ }^{4,5}$ that describe the measurements well. At $\sqrt{s}=8 \mathrm{TeV}$, the ATLAS and CMS measurements are in good agreement with each other and with the theory predictions, while there is a difference of about two standard deviations at $\sqrt{s}=7 \mathrm{TeV}$.

79 1.2. Differential $t \bar{t}$ cross-section measurements

80 The measurement of the single differential cross-sections for $t \bar{t}$ production allows

81 to perform even more stringent tests of the predictions of pQCD by exploring the

82 dynamics of the $t \bar{t}$ system. The ATLAS Collaboration has measured differential

83 cross-sections in the lepton+jets channel both in the resolved ${ }^{12,13}$ and boosted ${ }^{14}$

84 regimes.

85 The measurement of the normalized differential cross-sections for top-quark pair ${ }_{86}$ production $^{12}$ at $\sqrt{s}=7 \mathrm{TeV}$ has been performed as a function of several variables ${ }_{87}$ characterising the $t \bar{t}$ system: the transverse momentum of the top quark $\left(p_{\mathrm{T}}^{t}\right)$ and 


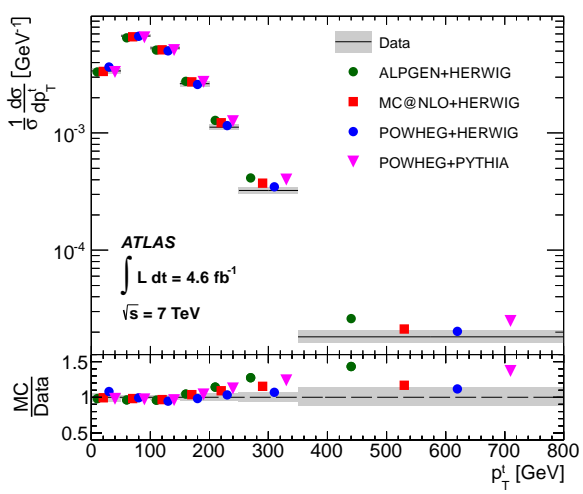

Fig. 2. Normalized differential cross-section as a function of the transverse momentum of the hadronically decaying top quark ${ }^{12}$ compared with different MC generator predictions. The gray band is the total uncertainties of the data. The lower part of the figure shows the ratio of the generator predictions to the data.

the invariant mass $\left(m_{t \bar{t}}\right)$, the transverse momentum $\left(p_{\mathrm{T}}^{t \bar{t}}\right)$ and the rapidity $\left(y_{t \bar{t}}\right)$ of the $t \bar{t}$ system. Events are selected in the lepton+jets channel, requiring exactly one lepton and at least four jets with at least one of the jets tagged as originating from a b-quark. Figure 2 shows the normalised differential cross-section as a function of the transverse momentum of the hadronically decaying top quark compared with the predictions of different Monte Carlo (MC) generators. The measurements are in fair agreement with the MC predictions except in the region of the top quark high transverse momentum where the data distributions are softer than the predictions. A similar measurement has been proformed by the CMS experiment and a similar behaviour is observed. A possible explanation of this difference between data and MC may be related to the gluon density of the proton.

The ATLAS Collaboration presented also the first measurement of the differential cross-sections in terms of various kinematic variables of the so called "pseudotop-quark" ${ }^{13}$, defined in terms of either reconstructed detector objects or stable particles, in order to achieve a better correspondence between detertor level and particle level measurements and a reduced dependence on the theoretical models. The cross-section is presented as a function of the transverse momentum and rapidity of both the hadronic and leptonic pseudo-top-quark as well as the transverse momentum, rapidity and invariant mass of the pseudo-top-quark pair system and compared with several MC models that implement NLO order or LO multi-leg matrix-element calculations. Figure 3 shows the results for the differential crosssection as a function of the $t \bar{t}$ pseudo-top-quark invariant mass $m\left(\hat{t}_{l} \hat{t}_{h}\right)$ and hadronic pseudo-top-quark $p_{\mathrm{T}}\left(\hat{t}_{h}\right)$. The comparison is performed with various LO multi-leg MC generators and various NLO MC generators. 
October 20 ,
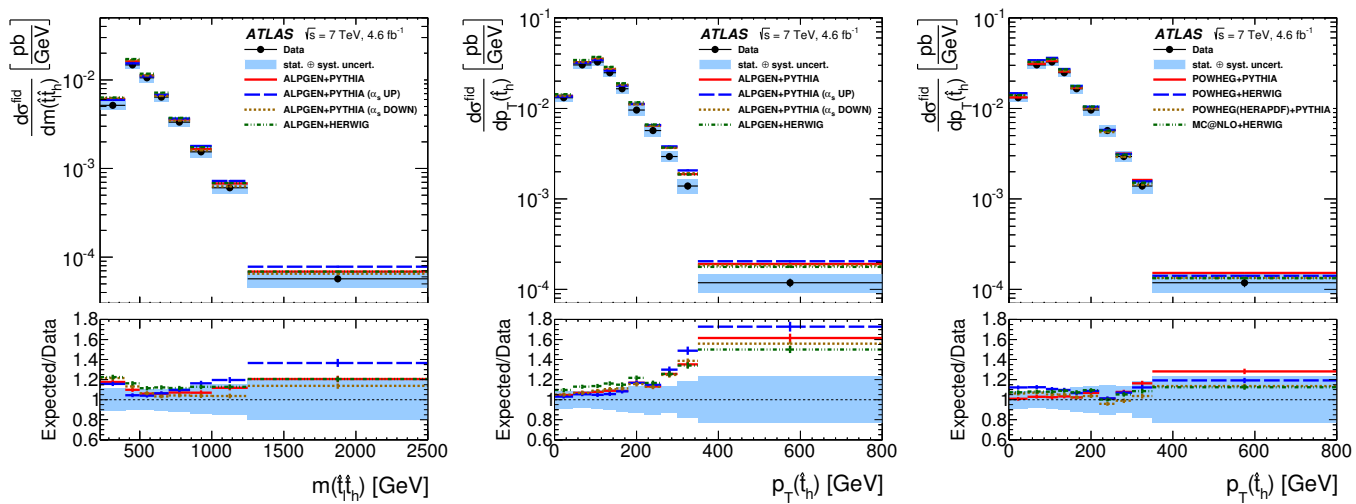

Fig. 3. (Left) Differential $t \bar{t}$ cross-section as a function of the $t \bar{t}$ pseudo-top-quark invariant mass $m\left(\hat{t}_{l} \hat{t}_{h}\right)$. (Middle) Differential $t \bar{t}$ cross-section as a function of the hadronic $t \bar{t}$ pseudo-top-quark transverse momentum $p_{\mathrm{T}}\left(\hat{t}_{h}\right)$. (Right) Differential $t \bar{t}$ cross-section as a function of the hadronic pseudo-top-quark $p_{\mathrm{T}}\left(\hat{t}_{h}\right)^{13}$. The data points are shown with a blue band which represents the total uncertainty (statistical and systematic) and compared with several LO multi-leg (middle) and NLO (right) MC generators.

The ALPGEN prediction ${ }^{15}$ do not describe correctly the cross-section as a function of $m\left(\hat{t}_{l} \hat{t}_{h}\right)$, in particular in the low mass region, and also in the high $p_{\mathrm{T}}\left(\hat{t}_{h}\right)$ region where a similar trend is observed as for the resolved results previously discussed. The NLO POWHEG(HERAPDF) + PYTHIA results ${ }^{16}$ provide the best description of all of the distributions.

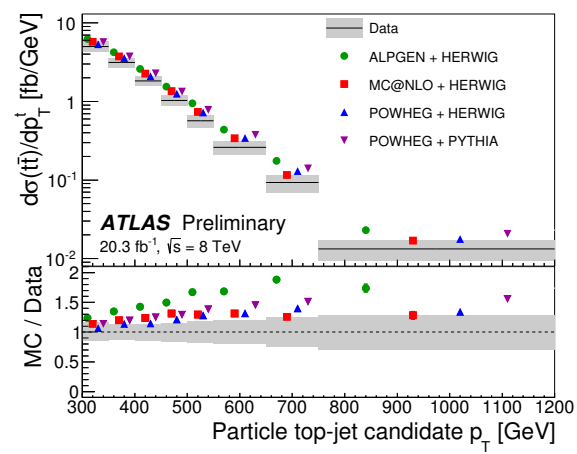

Fig. 4. Fiducial particle-level differential cross-section as a function of the transverse momentum of the hadronically decaying top quark compared with different MC generators. MC samples are normalized to the NNLO+NNLL inclusive cross-section ${ }^{14}$. The gray band is the total data uncertainties. The lower part of the figure shows the ratio of the generator predictions to the data. 
A similar conclusion, on the description of the distribution as a function of the top $p_{\mathrm{T}}$, is obtained for the measurement ${ }^{14}$ in the boosted regime, characterized by a large value of the transverse momentum of the produced top quarks. In this analysis the differential cross-section is presented in the fiducial and total phasespace as a function of the hadronically decaying top quark transverse momentum $p_{T}$ with a transverse momentum above $300 \mathrm{GeV}$ and shown in fig 4 . The predictions of NLO and LO matrix element combined with parton shower MC generators are found to generally overestimate the measured cross-sections. This measurment at $\sqrt{s}=8 \mathrm{TeV}$ has been performed by reconstructing the boosted top quarks with the anti- $k_{t}$ jet algoritm with the large radius parameter $R=1.0$, and a dedicated selection that exploits the rich content in the subjets of the fat jet originating from the boosted top quark that increases the selection efficiency at high $p_{\mathrm{T}}$ and extends the kinematics to the $\mathrm{TeV}$ scale. The dominant uncertainty in this measurement is due to the large-R jets energy scale.

\section{Single-top production}

Top quarks can be produced singly via electroweak, charged-current interactions. The single top cross-section in proton-proton collisions at $\sqrt{s}=8 \mathrm{TeV}$ is about half of that for top pair production mediated via the strong interaction. There are three relevant single top-quark subprocesses that are distinguished at LO by the virtuality of the exchanged $\mathrm{W}$ boson. At the LHC, the single top quark production is dominated by the t-channel exchange of a virtual $\mathrm{W}$ boson process $(\sim 80 \%)$,
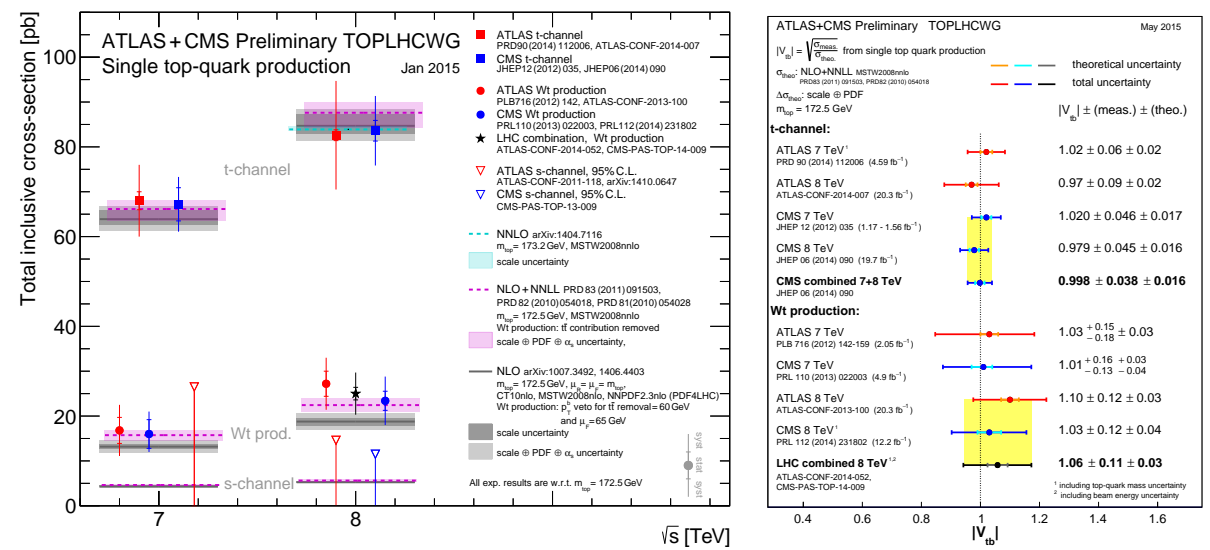

Fig. 5. (Left) Summary of ATLAS and CMS measurements of the single top production crosssection in various channel as a function of the centre-of-mass energy ${ }^{11}$. The measurement are ompared to theoretical calculation based on: NLO, NLO+NNLL and NNLO QCD predictions. (Right) Compilation of the ATLAS and CMS determination of the CKM matrix element $\left|V_{t b}\right|$ from single top quark measurements ${ }^{11}$. 

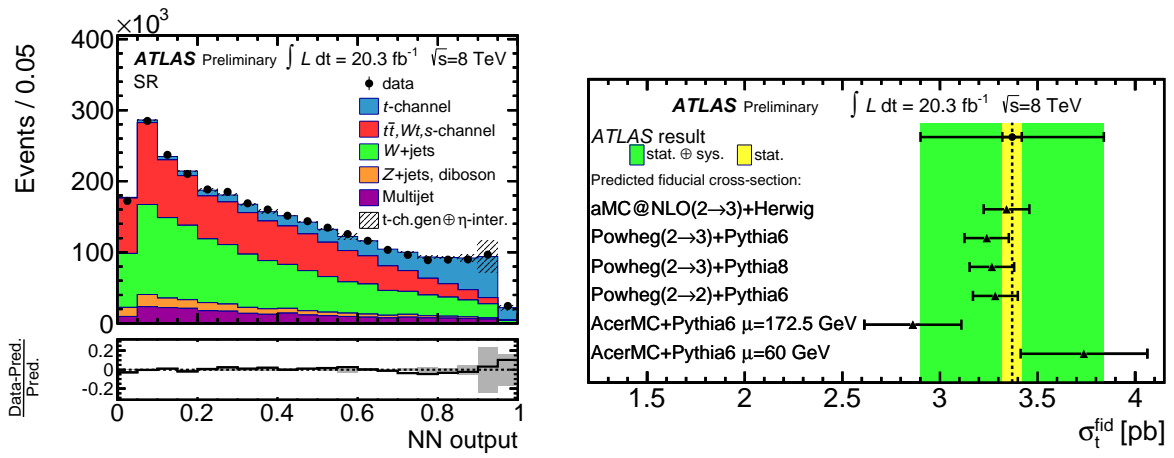

Fig. 6. (Left) Neural-network output-distribution. (Right) Predicted t-channel single top-quark fiducial cross-section for different LO and NLO MC generators together with the measured value ${ }^{21}$. The uncertainty on the prediction consists of the scale uncertainty and the uncertainty on the PDFs.

followed by the Wt production ( 15\%) while for the s-channel production only upper limits are given by the LHC experiments. The table below summarizes the theoretical predictions at NLO+NNLL for the crosse sections at 7, 8 and $14 \mathrm{TeV}$.

Table 1. SM predicted cross-sections

\begin{tabular}{cccc}
\hline & t-channel ${ }^{18}$ & Wt-channel ${ }^{19}$ & s-channel 20 \\
\hline $7 \mathrm{TeV}$ & $64.57_{-1.74}^{+2.63} \mathrm{pb}$ & $15.74_{-1.21}^{+1.17} \mathrm{pb}$ & $4.63_{-0.18}^{+0.20} \mathrm{pb}$ \\
$8 \mathrm{TeV}$ & $87.76_{-1.91}^{+3.44} \mathrm{pb}$ & $22.37 \pm 1.52 \mathrm{pb}$ & $5.61 \pm 0.22 \mathrm{pb}$ \\
$14 \mathrm{TeV}$ & $243 \pm 9 \mathrm{pb}$ & $83.6_{-6.8}^{+5.0} \mathrm{pb}$ & $11.9 \pm 0.6 \mathrm{pb}$ \\
\hline
\end{tabular}

The single top-quark cross-section is proportional to the square of the $\mathrm{W}$-t-b coupling at the production vertex. therefore, the measurement of the production cross-section constrains the absolute value of the quark-mixing matrix element ${ }^{17}$ $\left|V_{t b}\right|$. It is then possible to extract $\left|V_{t b}\right|$ by dividing the measured inclusive single top cross-section by the corresponding SM prediction. The figure 5 left shows a summary of the present single top production cross-section in the various channels ${ }^{21-23}$ compared with the NLO+NNLL theoretical predictions available at the centre-ofmas energy of 7 and $8 \mathrm{TeV}$.

The most precise ATLAS results for the t-channel production ${ }^{21}$ are presented in terms of fiducial and total cross-sections at $\sqrt{s}=8 \mathrm{TeV}$ with events selected in the lepton+jets channel. Events are selected by requiring exactly two jets with one of the jets being b-tagged and the signal component is enhanced by the use of a neural-network discriminant based on the final-state observables. Figure 6 left shows the neural-network (NN) output-distribution for the combined electron and muon channel and figure 6 right shows a comparison of the fiducial cross-section 
156

Fig. 7. Differential cross-section as a function of $p_{\mathrm{T}}$ and $y$ compared to the QCD NLO calculations $^{24}$.

with LO and NLO generators.

Using various MC generators, the fiducial cross-section can be extrapolated to the full phase-space and can be compared to the theoretical prediction.The measured value for the total cross-section in the t-channel is

$$
\sigma_{t}=82.6 \pm 1.2(\text { stat. }) \pm 11.4(\text { syst. }) \pm 3.1(\text { pdf }) \pm 2.3(\text { lumi }) \mathrm{pb}
$$

in good agreement with the prediction available and reported in table 1. A compilation of the relevant CKM matrix element $\left|V_{t b}\right|$ values determined in the $t^{21}$ and $\mathrm{Wt}^{22}$ channels is presented in figure 5 right.

The relative large cross-section for single top production in the t-channel allows also the measurement of differential cross-section ${ }^{24}$. The ATLAS collaboration has measured single top differential cross-sections using the complete data sample at $\sqrt{s}=7 \mathrm{TeV}$. The analysis strategy is very similar to the one described for the fiducial and total cross-section at $\sqrt{s}=8 \mathrm{TeV}$, applying a cut on the neural network output, a high purity region is defined to measure the differential cross-section as a function of the top and antitop transverse momentum and rapidity. In figure 7 the measured spectrum as a function of the $p_{\mathrm{T}}$ is shown and compared to NLO prediction that provide a good description of the measurement.
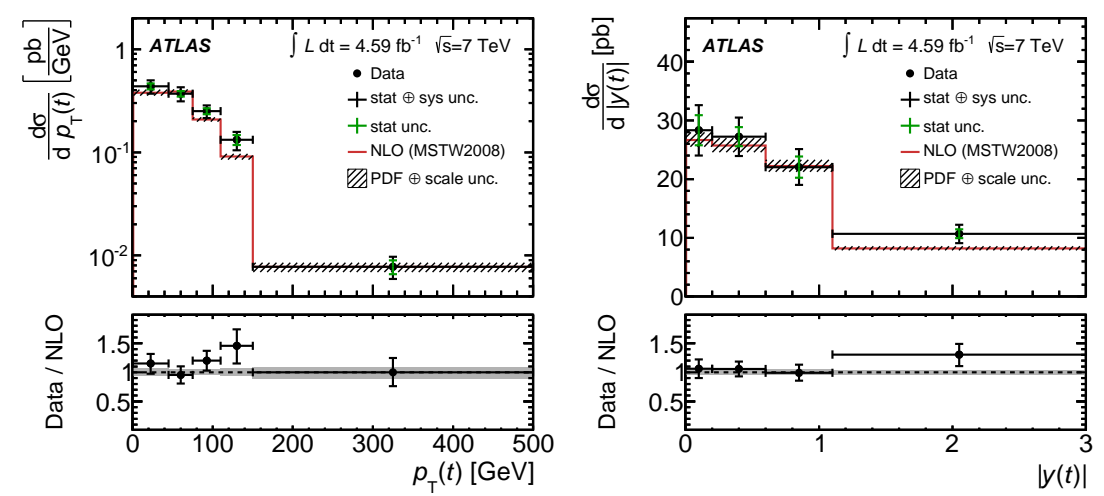

\section{Top properties}

In addition to the studies of the production mechanism for top pair and single top production, there is at LHC an intense activity devoted to the study of the properties of the top quark such as the top mass, the top charge asymmetry and spin correlations. 
October 20,

ws-ijmpcs*_*Capua.v3
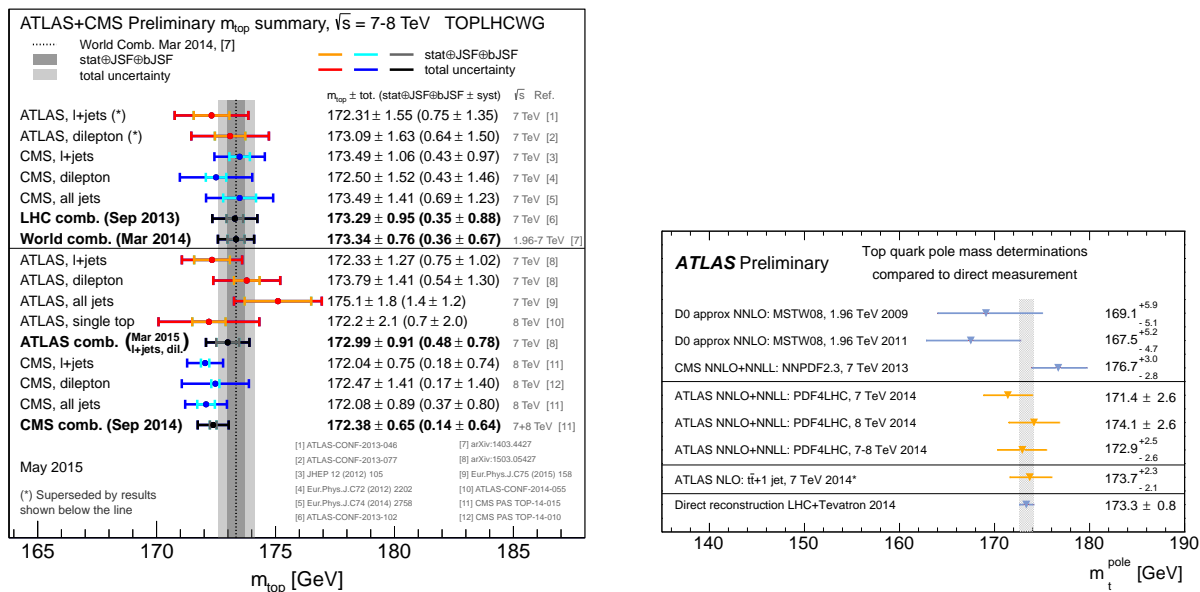

Fig. 8. (Left) Summary of the ATLAS and CMS direct $m_{t o p}$ measurements. The results are compared with the LHC and Tevatron+LHC $m_{t o p}$ combinations. For each measurement, the statistical uncertainty, the jet scale factor and b-jet scale factor contributions as well as the sum of the remaining uncertainties are reported separately. The results below the line are results produced after the LHC and Tevatron+LHC combinations were performed. (Right) Summary of the measurements of the top quark pole mass, compared to direct measurements.Taken from Ref. 11.

\section{Top mass measurements}

Various properties of the top quark have been measured, the most precisely measured quantity being the top quark mass. It is an important parameter of the SM via its large mass and a precise measurement is an important input to fits of global electroweak parameters that help to test the internal consistency of the SM.

The measurement of the top quark mass as the invariant mass of the top quark decay products has been performed using various techniques. In ATLAS analyses, the template method is commonly used. In particular, this method is adopted in a $t \bar{t}$ dedicated measurement ${ }^{25}$ performed with $\sqrt{s}=7 \mathrm{TeV}$ data in the dilepton and lepton+jets channels and their combination. This measurement represents the most precise ATLAS result on the top quark mass determination and results in a top mass of $m_{\text {top }}=172.99 \pm 0.91 \mathrm{GeV}$. Another important ATLAS result come from the single top t-channel measurement ${ }^{26}$ at $8 \mathrm{TeV}$. The analysis presents the first $m_{\text {top }}$ measurement in topologies of t-channel single top enriched with $t \bar{t}$ contribution are used. This measurement yielded the value $m_{t o p}=172.2 \pm 2.1 \mathrm{GeV}$.

Thanks to the improved performance, the precise signal MC modelling and new measurement techniques, the top mass measurements becaming increasingly precise. The first world combination of $m_{t o p}$ was carried out by the LHC and TeVatron experiments $^{27}$ and the following world average was obtained:

$$
m_{\text {top }}=173.34 \pm 0.76 \mathrm{GeV} \text {. }
$$




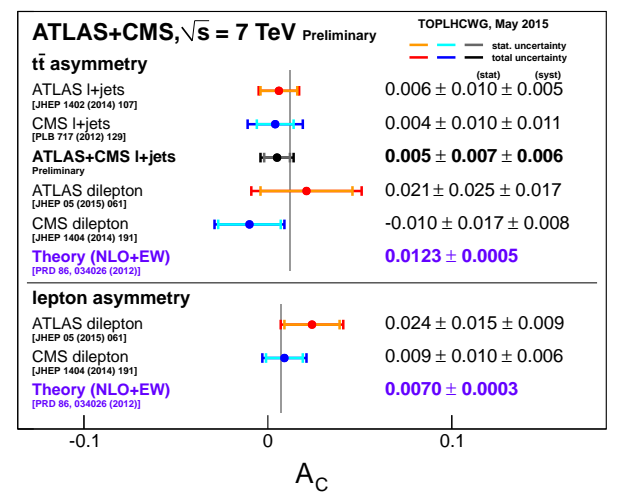

Fig. 9. Summary of the charge asymmetry measurements of the ATLAS and CMS results at $7 \mathrm{TeV}$ showing both the $t \bar{t}$-based and lepton-based asymmetry measurements, compared to the respective theory predictions ${ }^{11}$.

Figure 8 shows a summary of the top quark mass measurements entering the world combination $^{27}$.

It is also important to measure $m_{\text {top }}$ using alternative approach like the comparison of NNLO+NNLL QCD calculations to the inclusive $t \bar{t}$ production cross-section measurements ${ }^{2}$. Since this QCD calculation uses the pole mass scheme, the top $m_{\text {pole }}$ can be determined. Figure 8 right summarizes the top quark pole mass measurements obtained with the $t \bar{t}$ analisys in the dileptone channel ${ }^{2}$ already described and the recent measurement performed with $t \bar{t}+1$-jet events ${ }^{28}$ and compare the results to the direct measurements, as it is visible all the results are in good agreement. Although the precision of the results of $\sim 1.5 \%$ is not competitive with the direct $m_{\text {top }}$ measurements, the results are affected by different systematic uncertainties and allows for extractions of $m_{t o p}$ in theoretically well-defined mass schemes.

\section{Top charge asymmetry measurement}

In SM $t \bar{t}$ production the asymmetry is introduced by the higher order corrections, starting with the NLO in QCD contributions to the differential cross-sections. The asymmetry predicted in the SM is small and positive, implying that the top quark is preferentially emitted in the direction of the quark in the initial state. At the LHC, the contributions to the asymmetries are predominantly from $q \bar{q}$ and $q g$-initiated $t \bar{t}$ events. In the $q \bar{q}$ case, the quark is in most cases a valence quark whereas the antiquark is from the sea. The asymmetry translates to a higher boost along the beam direction for the $t$ quark than for the $\bar{t}$ quark. The rapidity distribution reflects this effect.

The ATLAS Collaboration presented results at $\sqrt{s}=7 \mathrm{TeV}$ performed in the dilepton $^{29}$ and $1+$ jets $^{30}$ channels. The dilepton measurement shows results in terms 


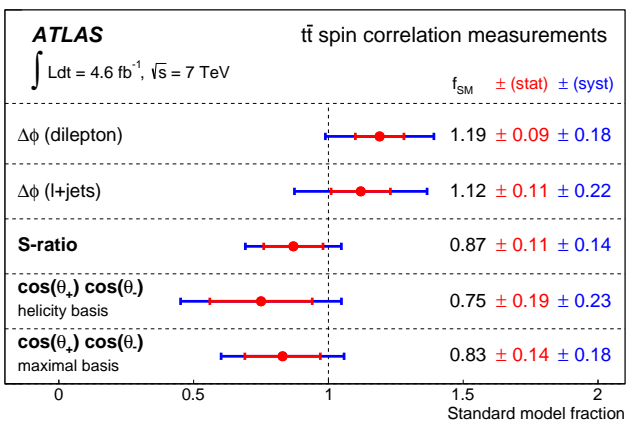

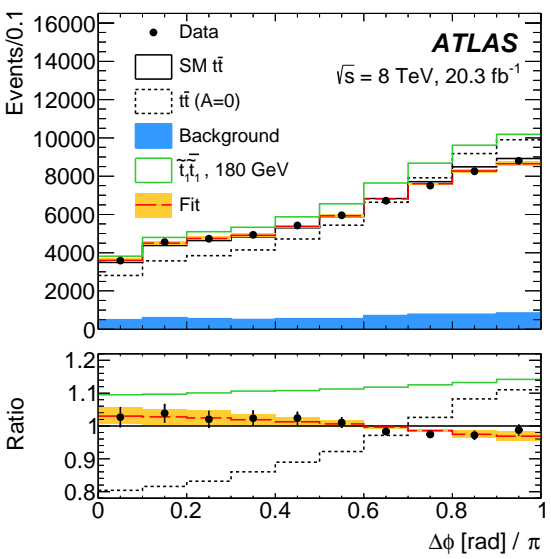

Fig. 10. (Left) Summary of the measurements of the fraction of $t \bar{t}$ events corresponding to the SM spin correlation hypothesis, $f_{S M}$, in the dilepton final state, using four spin correlation observables and in the lepton+jets channel. Dashed vertical line indicates the SM prediction ${ }^{31}$. (Right) Reconstructed $\Delta \phi$ distribution for the sum of the three dilepton channels ${ }^{32}$.

of $t \bar{t}$ charge asymmetry $A_{C}^{t \bar{t}}$ and leptons forward-backward asymmetry $A_{C}^{l l}$ :

$$
A_{C}^{t \bar{t}}=\frac{N(\Delta|y|>0)-N(\Delta|y|<0)}{N(\Delta|y|>0)+N(\Delta|y|<0)} \quad A_{C}^{l l}=\frac{N(\Delta|\eta|>0)-N(\Delta|\eta|<0)}{N(\Delta|\eta|>0)+N(\Delta|\eta|<0)}
$$

where $y$ and $\eta$ are the $t \bar{t}$ rapidity and lepton-lepton pseudorapidity respectively. The lepton+jets measurements are presented on terms of the $A_{C}^{t \bar{t}}$ asymmetry only.

The results are summarized in Figure 9 and are compatible with SM predictions.

\section{Top Spin correlations measurement}

In the SM, $t \bar{t}$ pairs are produced essentially unpolarized at hadron colliders. Nonetheless, the top quark and the antitop quark spins are correlated and the spin information transferred to the decay products and can be measured directly via their angular distributions.

Observables in the laboratory frame and in different top quark spin quantization bases can be used to measure the coefficent $f_{S M}$, which is connected to the number of events spin correlation as predicted by the SM. The coefficient is related to the spin correlation strength $A_{\text {helicity }}$ defined as the differences between events where the top quark spins is parallel and antiparallel with respect to a spin quantization axis, divided by the total number of events.

In the ATLAS measurement, performed at $\sqrt{s}=7 \mathrm{TeV}$ selecting dilepton and lepton + jet events ${ }^{31}$, the spin correlations are measured using the azimutal difference $\Delta \phi$ between the lepton and one of the final-state jets or between the two leptons, respectively. The spin correlation strenght obtained is $A_{\text {helicity }}=0.36 \pm 10$. Figure 10 
October 20,

ws-ijmpcs*_*Capua.v3

left shows the results obtained in terms of $f_{S M}$. In the figure are also presented additional measurements using observables which are different linear combinations of components in the spin density matrix of $t \bar{t}$ production. In particular, the ratio of the squares of matrix elements in the dileptonic channel is also measured. All the results are in good agreement with the SM prediction at NLO.

A new measurement of the spin correlations at $8 \mathrm{TeV}^{32}$, with $t \bar{t}$ dilepton events, uses the difference in the azimuthal angle between the two charged leptons in the laboratory frame. The spin correlation strenght obtained is $A_{\text {helicity }}=0.38 \pm 0.04$. In this analysis top squarks with masses between the top mass and $191 \mathrm{GeV}$ are excluded at the $95 \%$ C.L.

\section{References}

1. Yuan, C.P. arXiv:hep-ph/9809536 (1998).

2. ATLAS Collaboration, Eur. Phys. J., C74, 3109 (2014).

3. ATLAS Collaboration, Phys. Rev. D, 91, 112013 (2015).

4. M. Czakon, P. Fiedler, A. Mitov, Phys. Rev. Lett., 110, 252004 (2013).

5. M. Czakon, A. Mitov, Comput. Phys. Commun., 185, 2930 (2014).

6. M. Beneke et al., Nucl. Phys. B 855, 695 (2012).

7. M. Cacciari and others, Phys. Lett. B, 710, 612 (2012).

8. P. Brnreuther et al., Phys. Rev. Lett. 109, 132001 (2012).

9. M. Czakon, A. Mitov, J. High Energy Phys. 1212, 054 (2012).

10. M. Czakon, A. Mitov, J. High Energy Phys. 1301, 080 (2013).

11.

https://atlas.web.cern.ch/Atlas/GROUPS/PHYSICS/CombinedSummaryPlots/TOP/

12. ATLAS Collaboration, Phys. Rev. D, 90, 072004 (2014).

13. ATLAS Collaboration, JHEP 1506, 100 (2015).

14. ATLAS Collaboration, ATLAS-CONF-2014-057 (2014).

15. M. L. Mangano, M. Moretti, F. Piccinini, R. Pittau, A. Polosa, JHEP, 07, 001 (2003).

16. H1 and ZEUS Collaborations, JHEP, bf 01, 109 (2010).

17. N. Cabibbo, Phys. Rev. Lett., 10 (1963);

M. Kobayashi and T. Maskawa, Prog. Theor. Phys., 49 (1973).

18. N. Kidonakis, Phys. Rev. D, 83, 091503 (2011).

19. N. Kidonakis, Phys. Rev. D, 82 , 054018 (2010).

20. N. Kidonakis, Phys. Rev. D, 81, 054028 (2010).

21. ATLAS Collaboration, Phys. Rev. D, 90, 112006 (2014).

22. ATLAS Collaboration, ATLAS-CONF-2013-100 (2013).

23. ATLAS Collaboration, Phys. Lett. B, 740, 118 (2015).

24. ATLAS Collaboration, Phys. Rev. D, 90, 112006 (2014).

25. ATLAS Collaboration, arXiv:1503.05427 (2015).

26. ATLAS Collaboration, arXiv:1507.01769 (2015).

27. The ATLAS, CDF, CMS and D0 Collaborations, arXiv: 1403.4427 (2014).

28. ATLAS Collaboration, ATLAS-CONF-2014-053 (2014).

29. ATLAS Collaboration, JHEP, 05, 061 (2015).

30. ATLAS Collaboration, JHEP, 02, 107 (2014).

31. ATLAS Collaboration, Phys. Rev. D, 90, 112016 (2014).

32. ATLAS Collaboration, Phys. Rev. Lett. 114, 142001 (2015). 